\title{
Histogram-Based Quantitative Evaluation of Endobronchial Ultrasonography Images of Peripheral Pulmonary Lesion
}

\author{
Kei Morikawa $^{a}$ Noriaki Kurimoto $^{\mathrm{b}} \quad$ Takeo Inoue $^{\mathrm{a}} \quad$ Masamichi Mineshita $^{\mathrm{a}}$ \\ Teruomi Miyazawa ${ }^{a}$

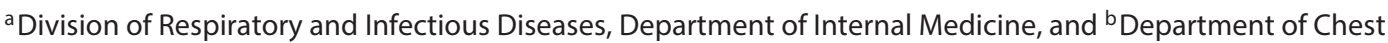 \\ Surgery, St. Marianna University School of Medicine, Kawasaki, Japan
}

\author{
Key Words \\ Bronchoscopy · Endobronchial ultrasonography . \\ Lung cancer imaging
}

\begin{abstract}
Background: Endobronchial ultrasonography using a guide sheath (EBUS-GS) is an increasingly common bronchoscopic technique, but currently, no methods have been established to quantitatively evaluate EBUS images of peripheral pulmonary lesions. Objectives: The purpose of this study was to evaluate whether histogram data collected from EBUS-GS images can contribute to the diagnosis of lung cancer. Methods: Histogram-based analyses focusing on the brightness of EBUS images were retrospectively conducted: 60 patients (38 lung cancer; 22 inflammatory diseases), with clear EBUS images were included. For each patient, a 400-pixel region of interest was selected, typically located at a 3- to 5-mm radius from the probe, from recorded EBUS images during bronchoscopy. Histogram height, width, height/width ratio, standard deviation, kurtosis and skewness were investigated as diagnostic indicators. Results: Median histogram height, width, height/width ratio and standard deviation were significantly different between lung cancer and benign lesions (all $p<0.01$ ). With a cutoff value for standard deviation of 10.5, lung cancer could be diagnosed with an accuracy of $81.7 \%$. Other characteristics investigated were inferior when compared to histogram standard deviation.
\end{abstract}

Conclusions: Histogram standard deviation appears to be the most useful characteristic for diagnosing lung cancer using EBUS images.

C) 2015 S. Karger AG, Basel

\section{Introduction}

In 2002, Kurimoto et al. [1] found that endobronchial ultrasonography (EBUS) images of peripheral pulmonary lesions could be visually classified into 3 types and 6 subtypes, and concluded that the images helped to differentiate between benign and malignant lesions. EBUS using a guide sheath (EBUS-GS) was subsequently established [2] and is rapidly gaining acceptance due to its excellent diagnostic yield. EBUS-GS, in which an ultrasound probe contained in a guide sheath is inserted into an access bronchus previously identified on chest computed tomography (CT) or a bronchial navigation system, could clearly visualize lesions undetectable on fluoroscopy. Lesions are brushed or biopsied via the guide sheath that has been left in position. Hence repeated interventions are possible at the same site, with the guide sheath also playing a hemostatic role.

Although CT images suggest malignancy, bronchoscopic sampling occasionally does not provide a pathological diagnosis. Therefore, analyzing EBUS images can provide additional information. The qualitative analysis

\section{KARGER 125}

(c) 2015 S. Karger AG, Base

$0025-7931 / 15 / 0892-0148 \$ 39.50 / 0$ 


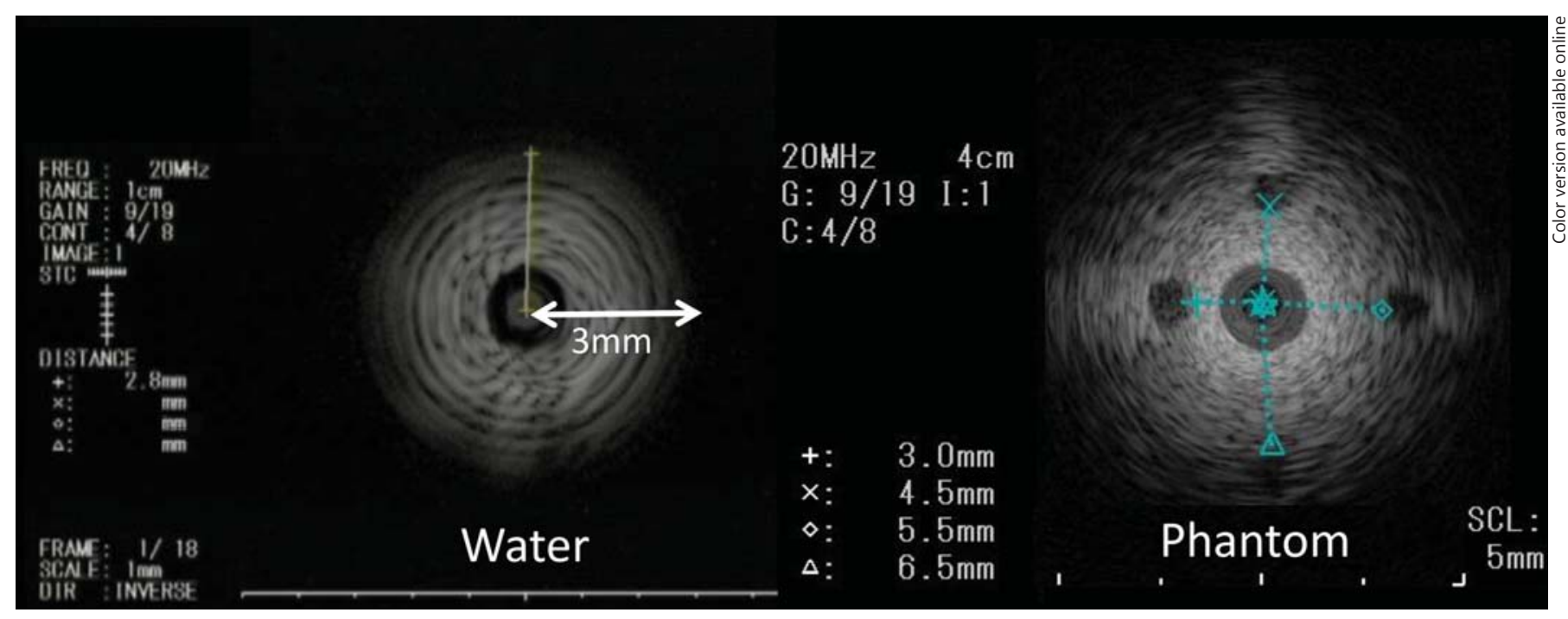

Fig. 1. Locations $3 \mathrm{~mm}$ from the center were unsuitable as an ROI for analysis due to artifacts caused by the ultrasound probe cover (left). Locations further than $5 \mathrm{~mm}$ from the center were also unsuitable due to attenuation of ultrasound signals (right).

of EBUS images shows that a homogeneous pattern suggests either an inflammatory disease or well-differentiated adenocarcinoma while a heterogeneous pattern suggests poorly differentiated carcinoma. In gastrointestinal and gynecological diseases, quantitative analyses of ultrasonography images have contributed to diagnosis [3-5]; however, there are no reports concerning quantitative analysis of EBUS images for peripheral pulmonary lesions. Therefore, we identified the need to establish a method to quantitatively analyze EBUS images since heterogeneity is difficult to predict with CT imaging alone.

\section{Material and Methods}

This study was performed at the Departments of Chest Surgery and of Internal Medicine, Division of Respiratory and Infectious Diseases Medicine of St. Marianna University School of Medicine Hospital, with ethics approval (Human Research and Ethics Committee approval reference No. 2179).

Among the patients who underwent bronchoscopy at our department from 2008 to 2012, 38 lung cancer patients and 22 inflammatory disease patients with clear images produced by EBUSGS were retrospectively analyzed. We used $92.3 \%$ (60/65 cases) of captured EBUS images for analyzing, while 5 images were unsuitable due to the size of the lesion or unclear images. Lung cancer was pathologically confirmed via specimens obtained by bronchoscopic means or at subsequent surgery. Patients were deemed to have an inflammatory disease if findings were benign by bronchoscopic sampling, surgical resected tissue or radiological opacities that diminished or disappeared on follow-up CT imaging 6 months later.

Histogram-Based Evaluation of EBUS-GS Image
Bronchoscopy was performed, with the patients sedated by midazolam, using primarily a flexible bronchoscope (BF-P260F or BF1T260; Olympus, Tokyo, Japan). Imaging of peripheral pulmonary lesions was performed with a miniature ultrasound probe (UM-S2017S, UM-S20-20R, Olympus) and guide sheath (K-201 kit, Olympus). EBUS images were rendered with an endoscopic ultrasound processor (Endoscopic Ultrasound Center; EU-M2000, Olympus). Identical gain and dynamic range (contrast) settings were used for all patients (displayed $9 / 19$ in gain, 4/8 in contrast). Images were obtained as still images and analyzed using Image J software (version 1.47, bundled with 64-bit Java, Sun Microsystems).

\section{Preliminary Study of Ultrasound Probe with Water and Phantom}

Prior to EBUS image analysis, a preliminary investigation was conducted into the characteristics and imaging capabilities of the ultrasound probe using a phantom model submerged in water. While the probe was inserted into the premanufactured hole of the model, locations of $3 \mathrm{~mm}$ or closer to the center of the model were found to be unsuitable as regions of interest (ROIs) due to artifacts caused by the ultrasound probe cover (fig. 1). This artifact is clearly seen when the EBUS probe is in water. However, when the probe is also in the lesion, especially when the probe is not fit to the leading bronchus, the probe cover and other artifacts are subject to appear in the image.

Using a phantom model, regions further than $5 \mathrm{~mm}$ from the center were found to be unsuitable as ROIs because of attenuation of ultrasound signals (fig. 1). We concluded that lesions located within concentric circles of $3-5 \mathrm{~mm}$ from the center would be suitable as ROIs.

\section{ROI Selection}

The size and shape selected for ROIs must allow the generated histograms to be comparable and analyzed across all patients. Selecting the same number of pixels to facilitate ROI comparison, the 
Fig. 2. Six histogram features were used for analyzing: height (maximum pixels; this case shows 100), width (maximum-minimum gray scale value; this case shows 50 ), height/width ratio (pixels/gray scale value; this case shows $100 / 50=2$ ), and standard deviation of histogram (this case shows 8.767).

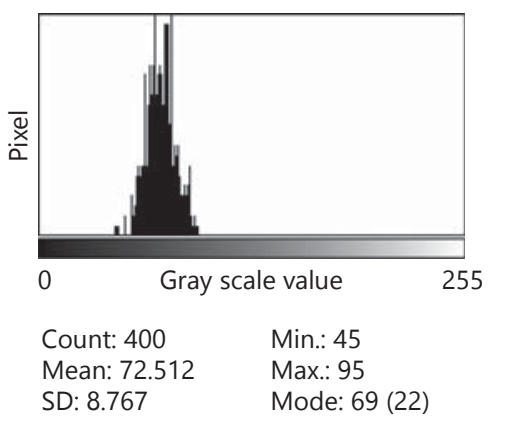

Six histogram features

- Height

- Width

- Height/width

- Standard deviation

- Kurtosis

- Skewness

Mean: 72.512

SD: $8.767 \quad$ Mode: 69 (22)

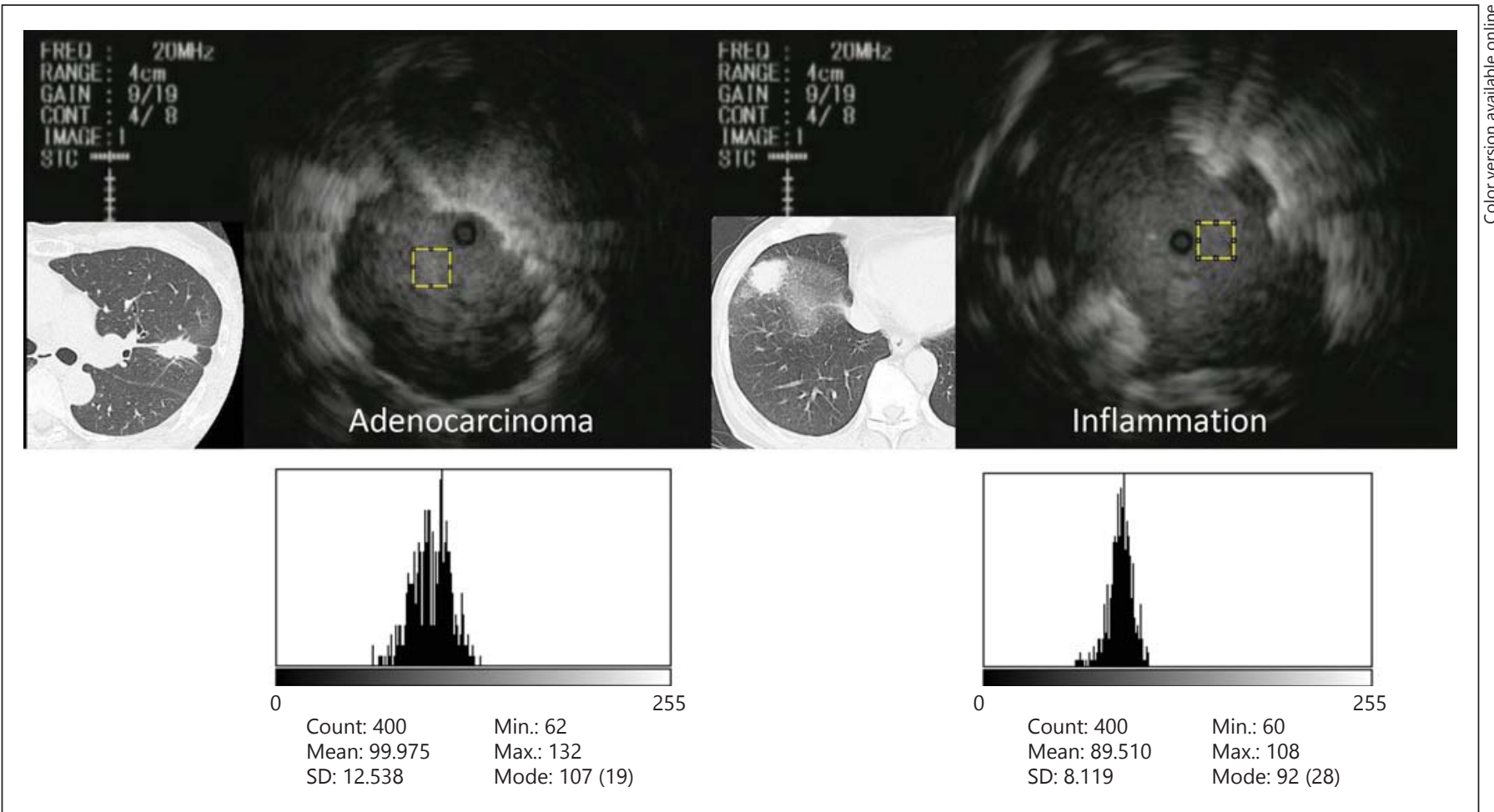

Fig. 3. B-mode image and histogram for typical cases of lung cancer (adenocarcinoma) and benign lesion (inflammation). In lung cancer, the histogram shape is wide and rough. In contrast, the histogram shape is sharp and tall in a benign lesion.

histogram shape analysis requires a size of 400 pixels. We generated square ROIs that were within concentric circles of 3-5 mm from the center of the probe and set ROIs in the center of the lesions to avoid borders and artifacts such as calcifications and vascular areas. We believe this choice allows for small lesions and lesions with uneven horizontal or vertical spread to be analyzed, and hence, it is more versatile.

\section{Histogram Analysis}

Six histogram features were used for analysis: height (the number of maximum pixels), width (maximum - minimum gray value), height/width ratio [the number of maximum pixels/ (maximum - minimum gray value)], standard deviation of histogram, kurtosis and skewness (fig. 2). The kurtosis and skewness of the histograms were calculated using Microsoft Excel 2010 with 32-bit (2010 Microsoft corporation) and Excel analysis software 2012 (2012 Social Survey Research Information Company, Japan). Mann-Whitney U tests were used to compare 6 histogram features between malignant and benign lesions.

Receiver-operating characteristic curves were plotted using Microsoft Excel 2010 to determine the optimal cutoff values for disease differentiation (fig. 4). B mode images and histograms of typical malignant and benign lesions are shown in figure 3 . 


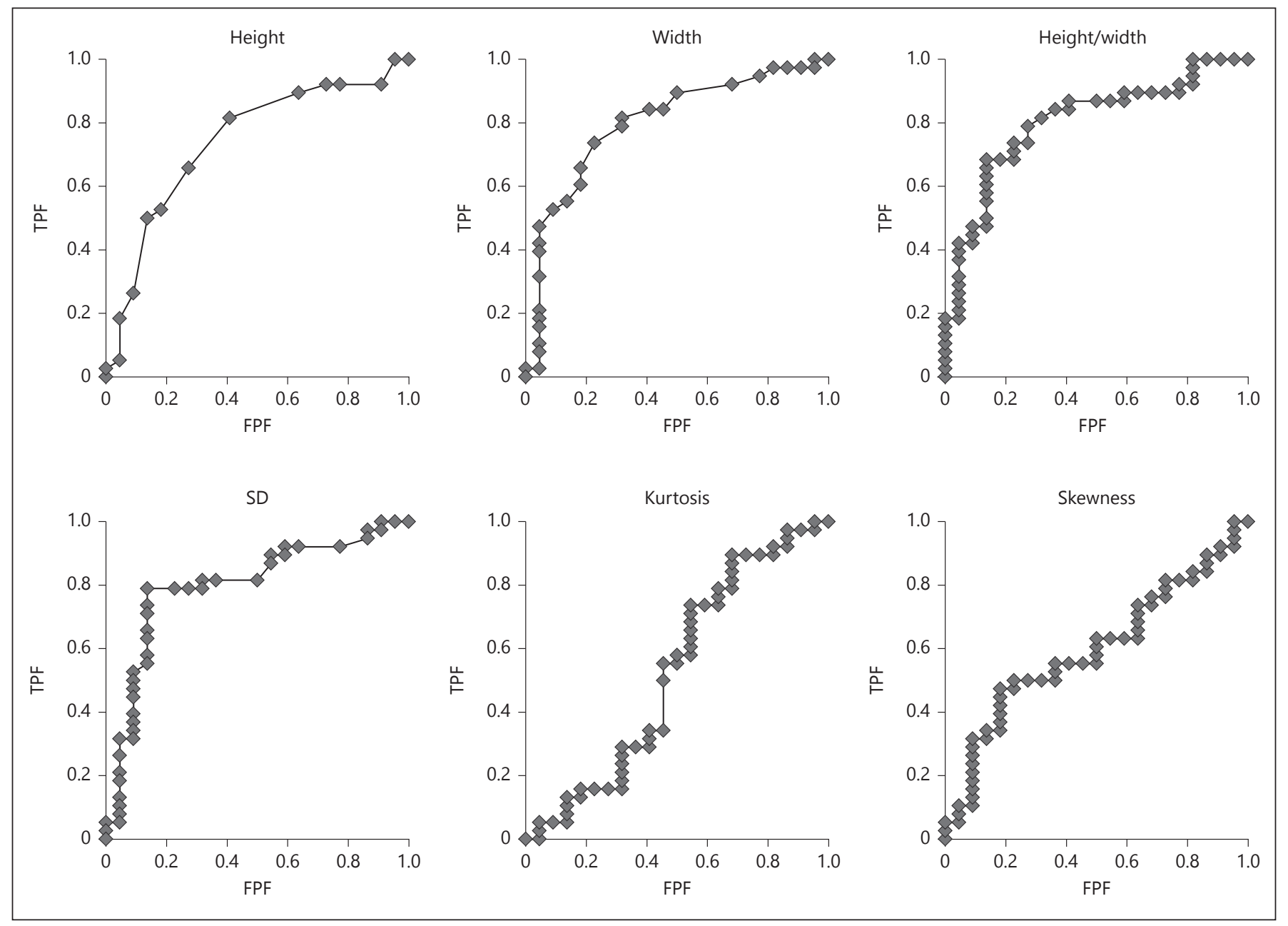

Fig. 4. A receiver-operating characteristic curve was made for each histogram parameter to determine a cutoff value. TPF $=$ True-positive fraction; FPF $=$ false-positive fraction.

\section{Results}

Of the 38 lung cancer patients, 19 had adenocarcinoma, 14 had squamous cell carcinoma, and 5 had small cell carcinoma. Most of the 22 inflammatory disease patients had nonspecific findings, although 3 cases that underwent bronchoscopic biopsy or surgery were given a pathological diagnosis of 1 organizing pneumonia and 2 tuberculomas. Of the 6 histogram parameters, height, width, height/width ratio and standard deviation were significantly different between malignant and benign lesions using statistical analysis. The median histogram height was 20 pixels (15-29) in the lung cancer group and 23 pixels (16-31) in the inflammation group. With a cutoff value of 21 pixels, cancer was diagnosed with a sensitivity of $65.8 \%$, specificity of $72.7 \%$ and an accuracy of $68.3 \%$. The median histogram width was 60 gray values (39-88) in the lung cancer group and 51 gray values $(38-76)$ in the inflammation group. With a cutoff value of 56 gray values, cancer could be diagnosed with a sensitivity of $73.7 \%$, specificity of $77.3 \%$ and an accuracy of $75.0 \%$. The median height/width ratio was 0.33 pixels/ gray value $(0.22-0.60)$ in the lung cancer group and 0.44 pixels/gray value (0.26-0.67) in the inflammation group. With a cutoff value of 0.3518 pixels/gray value, cancer could be diagnosed with a sensitivity of $68.4 \%$, specificity of $86.4 \%$, and an accuracy of $75.0 \%$. The median histogram standard deviation was $11.35(8.0-14.6)$ in the lung cancer group and $9.55(7.3-14.0)$ in the inflammation group. With a cutoff value of 10.5 , cancer was diagnosed with a sensitivity of $78.9 \%$, specificity of $86.4 \%$ and an accuracy of $81.7 \%$. Histogram kurtosis and skew- 
Table 1. Analyses of 6 histogram features

\begin{tabular}{lccc}
\hline & Malignant & Benign & p value \\
\hline $\begin{array}{l}\text { Number } \\
\text { Histogram feature }\end{array}$ & 38 & 22 & \\
$\quad$ Height, pixels & $20(15$ to 29$)$ & $23(16$ to 31$)$ & $<0.01$ \\
$\quad$ Width, gray scale values & $60(39$ to 88$)$ & $51(38$ to 76$)$ & $<0.01$ \\
$\quad$ Height/width, pixels/gray scale value & $0.33(0.22$ to 0.60$)$ & $0.44(0.26$ to 0.67$)$ & $<0.01$ \\
$\quad$ Standard deviation & $11.35(8.0$ to 14.6$)$ & $9.55(7.3$ to 14.0$)$ & $<0.01$ \\
Kurtosis & $-0.47(-1.21$ to 1.57$)$ & $-0.46(-1.22$ to 1.75$)$ & 0.7356 \\
$\quad$ Skewness & $0.64(0.02$ to 1.31$)$ & $0.75(0.24$ to 1.32$)$ & 0.2140 \\
\hline
\end{tabular}

Height, width, height/width ratio and standard deviation were significantly different in number between malignant and benign lesions by using Mann-Whitney U tests. Data are presented as medians with ranges in parentheses.

Table 2. Analyses of 6 features using receiver-operating characteristic curves

\begin{tabular}{lllllll}
\hline & $\begin{array}{l}\text { Cut point } \\
\text { value }\end{array}$ & $\begin{array}{l}\text { Sensitivity, } \\
\%\end{array}$ & $\begin{array}{l}\text { Specificity, } \\
\%\end{array}$ & $\begin{array}{l}\text { Positive predictive } \\
\text { value, } \%\end{array}$ & $\begin{array}{l}\text { Negative predictive } \\
\text { value, \% }\end{array}$ & $\begin{array}{l}\text { Accuracy, } \\
\%\end{array}$ \\
\hline Height & $<21.0$ & 65.8 & 72.7 & 73.5 & 55.2 & 68.3 \\
Width & $>56.0$ & 73.7 & 77.3 & 84.8 & 63.0 & 0.740 \\
Height/width & $<0.3518$ & 68.4 & 86.4 & 89.7 & 61.3 & 75.0 \\
Standard deviation & $>10.5$ & 78.9 & 86.4 & 90.9 & 70.4 & 0.801 \\
Kurtosis & $<0.3106$ & 73.7 & 45.5 & 70.0 & 50.0 & 0.801 \\
Skewness & $<0.5969$ & 50.0 & 77.3 & 79.2 & 47.2 & 63.3 \\
\hline
\end{tabular}

aROC $=$ Area under the receiver-operating characteristic curve.

ness were inferior when compared to the other histogram parameters. Diagnostic accuracies ranked in decreasing order were histogram standard deviation $(81.7 \%)$, width $(75.0 \%)$, height/width ratio $(75.0 \%)$, height (68.3\%), kurtosis (63.3\%) and skewness (60.0\%; tables 1, 2).

\section{Discussion}

Currently, no methods have been established for quantitatively analyzing peripheral pulmonary lesions; therefore, we devised a simple and versatile method for quantitatively evaluating EBUS B-mode images. To our knowledge, this is the first study to distinguish between benign and malignant lesions using histogram-based analysis of EBUS images for peripheral pulmonary lesions.

Histogram analysis is essentially analyzing gray scale images, and hence identical gain and contrast settings, which define the gray scale, were used for all patients. To avoid analyzing distorted images, we selected the clearest images taken when respiration was stable. The total lesion or part of the lesion can be selected as ROI. We also analyzed histogram features selecting total lesion of ROI. There were no significant differences in histogram width (lung cancer/inflammation, 118/120 gray values). In histogram height, there was a double-scored difference (lung cancer/inflammation, 300/165 pixels), and this difference might originate from the number of the lesion's ROI size (lung cancer/inflammation, 15,323/7,207 pixels). There was no significant difference in histogram standard deviation (lung cancer/inflammation, 22.73/22.66). However, the histogram mean gray value was significantly different (lung cancer/inflammation, 58/68 gray values) due to the amount of dark pixel areas in a lung cancer lesion that was reflected to the attenuation of the echo signal. Ideally, this comparison should be done using almost the same lesion size in both groups. However, it is difficult to collect lesions of similar sizes and is not always possible in clinical practice.

In this study, identical ROI sizes were used so that the center of the lesions could be compared. This highlighted 
differences in gray scale distribution across ROIs with identical number of pixels allowed for the identification of differences in gray scale distributions between cancer and inflammation. Although selecting a narrow part of the lesion as ROI has its disadvantages, i.e. does not reflect the entire lesion, broad ROIs were unnecessary because the goal was to compare speckle patterning, which is discussed below. Anechoic regions attributable to intralesional necrosis and blood vessels, while relevant to lesion evaluation, are artifacts on histogram analysis and should therefore be excluded.

Many studies have found EBUS/transbronchial needle aspiration with a convex probe useful for differentiating between benign and malignant lesions [6-11]. These studies included comparing lymph node size and shape. Lymph node echogenicity was another factor compared, and it tends to be heterogeneous in malignant lesions. Nguyen et al. [10], analyzing lymph node echogenicity with gray scale pattern analysis, concluded that wide gray scale values characterized malignancies. Although they studied lesions different from those in our study, it is interesting to note that our conclusion resembled theirs.

The grouping of bright, granular dots on ultrasound B-mode images is called speckle. Speckle is a pattern that is caused by interference from echo signals from many scatters smaller than the level of resolution. Image observers must remember that these bright dots do not have a one-to-one correspondence with histological features. Conversely, structures larger than the level of resolution are imaged as accurately shaped features. Most ROIs analyzed in this study contained image speckle. Visually homogeneous ultrasound images have very fine speckle, and visually heterogeneous images have rough speckle. With this in mind, it was possible to use histograms to differentiate between benign and malignant lesions with an accuracy of $81.7 \%$. In this study, histogram width, height/ width ratio and standard deviation resulted in more accurate diagnosis when compared with other parameters. Of the three, standard deviation better reflected histogram shape and resulted in a higher accuracy than the other two. We tried to combine different features for increasing the accuracy of diagnosis using logistic analysis. However, the results did not exceed an accuracy of a single parameter. A predictive value calculated using all parameters was a sensitivity of $68.18 \%$, specificity of $81.58 \%$ and an accuracy of $76.67 \%$.

Ultrasound B-mode images are obtained by log compressing radiofrequency signals in the device, meaning that most of the conventional ultrasound information is modified. Our procedure is designed to analyze modified image data and not directly analyze the ultrasound data itself. Although identical gain and contrast were used for all patients, there were still some limitations in standardization, and these included respiratory movements causing slight image distortion, degree of probe-lesion contact, movement at the site of probe-lesion contact along the long axis of the bronchus and other factors which impact on the brightness of the ultrasound image. The present image analysis seeks to differentiate lung cancer from inflammatory diseases. However, further basic studies in individual patients are needed to determine what kind of histological factors account for the homogeneity of lesions imaged with B-mode imaging. This is because cancerous lesions are varied and may range from poorly differentiated cancer that is pathologically highly necrotic to highly differentiated cancer with mutations, while patients with inflammatory diseases may exhibit either features of acute infection or fibrosis accompanying chronic infection. Even so, we devised a simple and versatile method for quantitatively evaluating these ultrasound images. These results have the potential to shorten bronchoscopic examination times in lung cancer by reducing the frequency of brushing and biopsy, and to observe pathologically negative cases in homogeneous EBUS image patterns which tend to result in inflammatory disease.

In the future, we aim to prospectively determine whether quantitatively evaluating image data in real-time during bronchoscopy can contribute to the pathological diagnostic work-up. The inclusion of radio frequency signal data in addition to B-mode image data will provide more detailed ultrasound information for analysis and can possibly make this technique a more robust diagnostic tool.

\section{Conclusion}

Quantitative analysis of histograms using EBUS images of peripheral pulmonary lesions appears to aid lung cancer diagnosis.

\section{Acknowledgments}

The authors thank Mr. Jason Tonge from St. Marianna University School of Medicine for reviewing the language of this paper. The authors also thank Dr. Shinobu Tatsunami of the Medical Statistics Department at St. Marianna University School of Medicine for valuable advice on the statistical analysis. 


\section{References}

$>1$ Kurimoto N, Murayama M, Yoshioka S, Nishisaka T, et al: Analysis of the internal structure of peripheral pulmonary lesions using endobronchial ultrasonography. Chest 2002;122:1887-1894.

-2 Kurimoto N, Miyazawa T, Okimasa S, Maeda A, Oiwa H, Miyazu Y, Murayama M: Endobronchial ultrasonography using a guide sheath increases the ability to diagnose peripheral pulmonary lesions endoscopically. Chest 2004;126:959-965.

-3 Fujii Y, Taniguchi N, Itoh K, Wang Y, Shigeta K, Ono T, Tsao JW, Kumasaki K, Itoh T: Quantitave processed images acquired by histogram-SNR imaging used to evaluate parenchymal heterogeneity in the liver. J Med Ultrasonics 2003;30:13-19.

4 Maeda K, Utsu M, Kihaile PE: Quantification of sonographic echogenicity with gray-level histogram width: a clinical tissue characterization. Ultrasound Med Biol 1998;24:225-234.
5 Maeda K, Utsu M, Yamamoto N, Serizawa M, Itoh T: Clinical tissue characterization with gray level histogram width in obstetrics and gynecology. Ultrasound Rev Obstet Gynecol 2002;2:124-128.

6 Nakajima T, Yasufuku K, Iyoda A, Yoshida S, Suzuki M, Sekine Y, Shibuya K, Hiroshima K, Nakatani Y, Fujisawa T: The evaluation of lymph node metastasis by endobronchial needle aspiration: crucial for selection of surgical candidates with metastatic lung tumors. J Thorac Cardiovasc Surg 2007;134:14851490.

7 Tagaya R, Kurimoto N, Osada H, Kobayashi A: Automatic objective diagnosis of lymph nodal disease by B-mode images from convex-type echobronchoscopy. Chest 2008;133: 137-142.

8 Fujiwara T, Yasufuku K, Nakajima T, Chiyo M, Yoshida S, Suzuki M, Shibuya K, Hiroshima K, Nakatani Y, Yoshino I: The utility of sonographic features during endobronchial ultrasound-guided transbronchial needle aspiration for lymph node staging in patients with lung cancer. Chest 2010;138:641-647.
9 Memoli JS, El-Bayoumi E, Pastis NJ, Tanner NT, Gomez M, Huggins JT, Onicescu G, Garrett-Mayer E, Armeson K, Taylor KK, Silvestri GA: Using endobronchial ultrasound features to predict lymph node metastasis in patients with lung cancer. Chest 2011;140: 1550-1556.

10 Nguyen P, Bashirzadeh F, Hundloe J, Salvado $O$, Dowson N, Ware R, Masters IB, Bhatt M, Kumar AR, Fielding D: Optical differentiation between malignant and benign lymphadenopathy by grey scale texture analysis of endobronchial ultrasound convex probe images. Chest 2012;141:709715

11 Schmid-Bindert G, Jiang H, Kähler G, Saur J, Henzler T, Wang H, Ren S, Zhou C, Pilz LR: Predicting malignancy in mediastinal lymph nodes by endobronchial ultrasound: a new ultrasound scoring system. Respirology 2012; 17:1190-1198. 